\title{
TOTAL CURVATURE OF BRANCHED MINIMAL SURFACES
}

\author{
YI FANG \\ (Communicated by Peter Li)
}

\begin{abstract}
An intrinsic, and much simpler, proof of a generalization of Jorge and Meeks' total curvature formula for complete minimal surfaces is given.
\end{abstract}

Let $X: M \rightarrow \mathbb{R}^{3}$ be a complete minimal surface with finite total curvature. Osserman proved that conformally $M=S_{k}-\left\{p_{1}, \cdots, p_{n}\right\}, n \geq 1$, where $S_{k}$ is a closed Riemann surface of genus $k$. See, for example, [4, Theorem 9.1, page 81]. Each $p_{i}$ corresponds to an end $E_{i}$ of $M$. Jorge and Meeks [2] proved that there is an integer $I_{i} \geq 1$ corresponding to $E_{i}$, such that the total curvature of $M$ is given by

$$
\int_{M} K d A=2 \pi\left(\chi(M)-\sum_{i=1}^{n} I_{i}\right),
$$

where $\chi(M)=2(1-k)-n$ is the Euler characteristic of $M$.

The proof of Jorge and Meeks involves a detailed study of the behaviour of the image $X(M)$ at each end. Since the Gauss curvature is an intrinsic quantity, it is natural to look for an intrinsic proof. In this note we give such an intrinsic, and much simpler, proof of a generalization of (1).

The Enneper-Weierstrass representation of a branched complete minimal surface of finite total curvature $X: M \rightarrow \mathbb{R}^{3}$ is given by

$$
X(p)=\operatorname{Re} \int_{p_{0}}^{p}\left(\frac{1}{2}\left(1-g^{2}\right), \frac{i}{2}\left(1+g^{2}\right), g\right) \eta,
$$

where $g: M=S_{k}-\left\{p_{1}, \cdots, p_{n}\right\} \rightarrow \mathbb{C} \cup\{\infty\}$ is a meromorphic function and $\eta$ is a holomorphic 1-form on $M$. Both $g$ and $\eta$ can be extended to $S_{k}$ as a meromorphic function and 1-form respectively; see [4, Theorem 9.1, page 81]. Note that since the proof given there only involves the neighbourhoods of the punctures $p_{i}$, it works for branched minimal surfaces as well.

Locally, $\eta=f(z) d z$, where $z=x+i y$. The metric induced by $X$ is given by

$$
d s^{2}=\Lambda^{2}\left(d x^{2}+d y^{2}\right),
$$

Received by the editors November 28, 1994.

1991 Mathematics Subject Classification. Primary 53A10.

Supported by Australian Research Council grant A69131962. 
where

$$
\Lambda=\frac{1}{2}|f|\left(1+|g|^{2}\right)
$$

see [4, pages 47,65]. From (4) it is clear that $q$ is a branch point only when $\eta$ vanishes at $q$. Hence all branch points are isolated, and if $\eta$ is a meromorphic 1-form on $S_{k}$, there is only a finite number of branch points.

Therefore, given $g$ and $\eta$ as above, define a metric with isolated degenerate points, $h_{i j}=\Lambda \delta_{i j}$ by (3) and (4). We can study the intrinsic geometry of the branched complete Riemannian manifold $(M, h)$ even though the mapping $X$ in (2) may not be well defined. When $X$ is well defined, it is a branched complete minimal surface.

Let $U_{i}$ be a disk coordinate neighbourhood of $p_{i}$ such that $z\left(p_{i}\right)=0$. Let $J_{i}$ be the order of $\Lambda$ at $p_{i}$, i.e., $J_{i}$ is an integer such that in $U_{i}$,

$$
\lim _{z \rightarrow 0}|z|^{J_{i}} \Lambda(z)=C_{i}>0,
$$

for $1 \leq i \leq n$. Since $(M, h)$ is complete, $J_{i} \geq 1$. In fact, if $X$ in (2) is well defined, it is well known that $J_{i} \geq 2$. See [3, page 135].

Suppose $q_{i}, 1 \leq i \leq m$, are branch points of $M$. Let $V_{i}$ be a disk coordinate neighbourhood of $q_{i}$ such that $z\left(q_{i}\right)=0$. Let $K_{i}>0$ be the branch order of $\Lambda$, i.e.,

$$
\lim _{z \rightarrow 0}|z|^{-K_{i}} \Lambda(z)=F_{i}>0, \quad \text { in } \quad V_{i}
$$

Theorem 1. The total curvature of $(M, h)$ is given by

$$
\int_{M} K d A=2 \pi\left(\chi(M)-\sum_{i=1}^{n}\left(J_{i}-1\right)+\sum_{i=1}^{m} K_{i}\right) .
$$

Proof. Let $R>0$ be such that $D_{R}^{i}:=\{|z|<R\} \subset U_{i}, 1 \leq i \leq n$ and $D_{R}^{i}:=\{|z|<$ $R\} \subset V_{i-n}, n+1 \leq i \leq n+m$. When $R$ is small enough, $D_{R}^{i} \cap D_{R}^{j}=\emptyset$ for $i \neq j$.

Let $M_{R}=M-\bigcup_{i=1}^{n+m} D_{R}^{i}$. By the Gauss-Bonnet formula, we have

$$
\int_{M_{R}} K d A+\sum_{i=1}^{n+m} \int_{\partial D_{R}^{i}} \kappa_{g} d s=2 \pi \chi\left(M_{R}\right)=2 \pi(\chi(M)-m) .
$$

If $g\left(p_{i}\right) \neq \infty$, then $\eta=z^{-J_{i}} f_{i}(z) d z$ where $f_{i}$ is a holomorphic function in $U_{i}$ and $f_{i}(0) \neq 0$. Write $z=r e^{i t}$. By Minding's formula, see [1, Volume I, pages 33-34], the geodesic curvature on $\partial D_{R}^{i}$ is given by

$$
\kappa_{g} \Lambda=-\frac{1}{R}+\frac{\partial \log \Lambda}{\partial \nu},
$$

where $\nu$ is the interior unit normal of $\partial D_{R}^{i}$. Now $\Lambda=1 / 2|z|^{-J_{i}}\left|f_{i}\right|\left(1+|g|^{2}\right)$, so

$$
\frac{\partial \log \Lambda}{\partial \nu}=-\frac{\partial \log \Lambda}{\partial r}=\frac{J_{i}}{r}-\frac{\partial \log \left|f_{i}\right|}{\partial r}-\frac{\partial \log \left(1+|g|^{2}\right)}{\partial r}
$$


and

$$
\int_{\partial D_{R}^{i}} \kappa_{g} d s=\int_{0}^{2 \pi} \kappa_{g} \Lambda R d t=\int_{0}^{2 \pi}\left(\frac{J_{i}-1}{R}-\frac{\partial \log \left|f_{i}\right|}{\partial r}-\frac{\partial \log \left(1+|g|^{2}\right)}{\partial r}\right) R d t .
$$

Since

$$
\int_{0}^{2 \pi} \frac{\partial \log \left|f_{i}\right|}{\partial r} R d t=\int_{D_{R}^{i}} \triangle\left(\log \left|f_{i}\right|\right) d x d y=0
$$

and $\partial \log \left(1+|g|^{2}\right) / \partial r$ is bounded, we have

$$
\lim _{R \rightarrow 0} \int_{\partial D_{R}^{i}} \kappa_{g} d s=2 \pi\left(J_{i}-1\right) .
$$

If $g\left(p_{i}\right)=\infty$, then $g=z^{-m_{i}} g_{i}(z), m_{i}>0$, and $\eta=z^{-J_{i}+2 m_{i}} f_{i}(z) d z$, where $h_{i}$ and $g_{i}$ are holomorphic functions in $U_{i}$ and $h_{i}(0) \neq 0, g_{i}(0) \neq 0$. Then

$$
\frac{\partial \log \Lambda}{\partial \nu}=-\frac{\partial \log \Lambda}{\partial r}=\frac{J_{i}-2 m_{i}}{r}-\frac{\partial \log \left|f_{i}\right|}{\partial r}-\frac{\partial \log \left(1+|g|^{2}\right)}{\partial r} .
$$

Since

$$
\begin{gathered}
\frac{\partial \log \left(1+|g|^{2}\right)}{\partial r}=\frac{1}{1+r^{-2 m_{i}}\left|g_{i}\right|^{2}}\left(-2 m_{i} r^{-2 m_{i}-1}\left|g_{i}\right|^{2}+r^{-2 m_{i}} \frac{\partial\left|g_{i}\right|^{2}}{\partial r}\right) \\
-\int_{0}^{2 \pi} \frac{\partial \log \left(1+|g|^{2}\right)}{\partial r} R d t=\int_{0}^{2 \pi} \frac{2 m_{i} R^{-2 m_{i}}\left|g_{i}\right|^{2}}{1+R^{-2 m_{i}}\left|g_{i}\right|^{2}} d t-\int_{0}^{2 \pi} \frac{R^{-2 m_{i} \frac{\partial\left|g_{i}\right|^{2}}{\partial r}}}{1+R^{-2 m_{i}}\left|g_{i}\right|^{2}} R d t \\
\rightarrow 4 m_{i} \pi \text { as } R \rightarrow 0 .
\end{gathered}
$$

We still have

$$
\lim _{R \rightarrow 0} \int_{\partial D_{R}^{i}} \kappa_{g} d s=2 \pi\left(J_{i}-1\right) .
$$

Similarly, for the branch points $q_{i}$, if $g\left(q_{i}\right) \neq \infty$, then $\eta=z^{K_{i}} f_{i}(z) d z$ where $f_{i}$ is a holomorphic function defined in $V_{i}$ and $f_{i}(0) \neq 0$. A similar calculation gives

$$
\int_{\partial D_{R}^{i+n}} \kappa_{g} d s=-\int_{0}^{2 \pi}\left(\frac{K_{i}+1}{R}+\frac{\partial \log \left|f_{i}\right|}{\partial r}+\frac{\partial \log \left(1+|g|^{2}\right)}{\partial r}\right) R d t .
$$

Hence

$$
\lim _{R \rightarrow 0} \int_{\partial D_{R}^{i+n}} \kappa_{g} d s=-2 \pi\left(K_{i}+1\right) .
$$

If $g\left(q_{i}\right)=\infty$, then $g(z)=z^{-m_{i}} g_{i}(z)$ and $\eta=z^{K_{i}+2 m_{i}} f_{i}(z)$; a similar calculation still gives us the same limit.

Note that

$$
\lim _{R \rightarrow 0} \int_{M_{R}} K d A=\int_{M} K d A .
$$

Letting $R \rightarrow 0$ in (6), we get (5). The proof is complete. 
Remark 1. If $X$ in (2) is well defined, then $h$ is induced by $X$. In this case, the order of $\Lambda$ at an end is invariant under a rotation in $\mathbb{R}^{3}$, and so we can assume that $g\left(p_{i}\right)=0$. By the Enneper-Weierstrass representation (2) and the definition of $I_{i}$ in [2], we see that $J_{i}-1=I_{i}$. Therefore, when $M$ is a regular minimal surface, (5) gives (1).

The calculation also works for boundary branch points. Let $M$ be a compact domain of a Riemann surface with a $C^{2}$ boundary $\Gamma=\partial M$. Suppose that $g$ and $\eta$ are a given meromorphic function and 1-form, respectively, and $h$ is the Riemannian metric with isolated degenerate points defined by (3) and (4). Let $q_{i} \in M(1 \leq i \leq m)$ be the interior branch points with branch order $K_{i}$ and $s_{i} \in M$ $(1 \leq i \leq n)$ be the boundary branch points with branch order $L_{i}$. Then

Theorem 2. The total curvature of $(M, h)$ is given by

$$
\int_{M} K d A=2 \pi\left(\chi(M)+\sum_{i=1}^{m} K_{i}\right)+\pi \sum_{i=1}^{n} L_{i}-\int_{\Gamma} \kappa_{g} d s .
$$

A sketch of the proof of (7) is as follows:

Define $D_{R}^{i}$ as before and $M_{R}=M-\bigcup_{i=1}^{n+m} D_{R}^{i}$. By the Gauss-Bonnet formula,

$$
\int_{M_{R}} K d A+\int_{\partial M_{R}} \kappa d s+\sum_{i=1}^{n}\left(\alpha_{R}^{i}+\beta_{R}^{i}\right)=2 \pi(\chi(M)-m),
$$

where $\alpha_{R}^{i}$ and $\beta_{R}^{i}$ are the exterior angles near the boundary branch points and

$$
\lim _{R \rightarrow 0} \alpha_{R}^{i}=\frac{\pi}{2}, \quad \lim _{R \rightarrow 0} \beta_{R}^{i}=\frac{\pi}{2} .
$$

Then (7) follows by

$$
\begin{aligned}
\lim _{R \rightarrow 0} \int_{\partial D_{R}^{i} \cap \partial M_{R}} \kappa d s & =\lim _{R \rightarrow 0} \int_{\epsilon_{R}^{i}}^{\delta_{R}^{i}}\left(\frac{-1}{R}-\frac{\partial \log \Lambda}{\partial r}\right) R d t=\lim _{R \rightarrow 0}\left(\epsilon_{R}^{i}-\delta_{R}^{i}\right)\left(1+L_{i}\right) \\
& =-\pi\left(1+L_{i}\right),
\end{aligned}
$$

for the boundary branch points.

Remark 2. If $X$ in (2) is well defined, then $X$ is a minimal surface and $h$ is induced by $X$. In this case, (7) is the same as the formula in [1, Volume II, page 128].

\section{REFERENCES}

1. U. Dierkes, S. Hidebrandt, A. Küster, O. Wohlrab., Minimal Surfaces, Vol. I \& II, SpringerVerlag, Berlin Heidelberg New York London Paris Tokyo Hong Kong Barcelona Budapest, 1992. MR 94c:49001b

2. L. P. Jorge and W. H. Meeks III., The topology of complete minimal surfaces of finite total Gauss curvature., Topology 22(2) (1983), 203-221. MR 84d:53006

3. H. B. Lawson, Jr., Lecture on Minimal Submanifolds, Publish or Perish, Inc., Berkeley, 1980. MR 82d:53035b

4. R. Osserman., A Survey of Minimal Surfaces, Dover Publications, Inc., New York, 1986. MR 87j:53012

Centre for Mathematics and its Applications, School of Mathematical Sciences, The Australian National University, Canberra, ACT 0200, Australia

E-mail address: yi@maths.anu.edu.au 